\title{
Glioblastoma multiforme em idosos: uma revisão sobre seu tratamento com ênfase na abordagem cirúrgica
}

\author{
Guilherme Lellis Badke', Alexandros Theodoros Panagopoulos², \\ Guilherme Brasileiro de Aguiar ${ }^{3}$, José Carlos Esteves Veiga ${ }^{4}$
}

Disciplina de Neurocirurgia, Irmandade da Santa Casa de Misericórdia de São Paulo, Faculdade de Ciências Médicas da Santa Casa de São Paulo (FCMSCSP), São Paulo, SP, Brasil.

\section{RESUMO}

O glioblastoma multiforme (GBM) é o tumor primário mais comum do sistema nervoso central (SNC). Em virtude do envelhecimento populacional, entre outros fatores, sua ocorrência em faixas etárias avançadas tem se mostrado crescente nas últimas décadas. Os objetivos da ressecção do GBM incluem: alívio do efeito de massa, citorredução tumoral, diagnóstico histopatológico, controle dos sintomas e melhoria nas taxas de sobrevivência. Por muito tempo acreditou-se que a idade seria fundamental na decisão pelo tratamento, embora alguns autores afirmem que a extensão da ressecção cirúrgica constitui o principal fator determinante do prognóstico, sobretudo para pacientes que foram eleitos para radioterapia e quimioterapia. A ressecção de mais de $98 \%$ do volume tumoral é capaz de interferir na sobrevivência, e ressecções menores proporcionaram sobrevivência semelhante à dos pacientes não abordados. O presente trabalho tem por objetivo realizar uma revisão da literatura atual a respeito do tratamento do GBM nos idosos, com enfoque especial na abordagem cirúrgica.

\section{PALAVRAS-CHAVE}

Gliobastoma, idoso, terapêutica, neurocirurgia.

\section{ABSTRACT}

Glioblastoma multiforme in the elderly: a review on their treatment with emphasis in the surgical approach

The glioblastoma multiforme (GBM) is the most common primary tumor of the central nervous system (CNS). Due to the aging population, among other factors, its occurrence in older age groups has shown increasing in recent decades. The goals of resection of GBM include: relieve mass effect, tumor debulking, histopathological diagnosis, symptom control and improvement in survival rates. For a long time it was believed that age would be crucial in deciding the treatment, although some authors state that the extent of surgical resection is the main determinant of prognosis, especially for patients who were elected to radiotherapy and chemotherapy. The resection of more than $98 \%$ of the tumor volume can interfere with the survival and minor resections provided similar to survival of patients not operated. This paper aims to review the current literature regarding the treatment of GBM in the elderly, with special focus on surgical approach.

\section{KEYWORD}

Gliobastoma, aged, therapeutics, neurosurgery.

1 Residente de Neurocirurgia da Irmandade da Santa Casa de Misericórdia de São Paulo (ISCMSP), São Paulo, SP, Brasil.

2 Neurocirurgião do Serviço de Neurocirurgia, ISCMSP, e professor instrutor da Faculdade de Ciências Médicas da Santa Casa de São Paulo (FCMSCSP), São Paulo, SP, Brasil.

3 Neurocirurgião do Serviço de Neurocirurgia, ISCMSP, São Paulo, SP, Brasil.

4 Neurocirurgião, chefe do Serviço de Neurocirurgia, ISCMSP, e professor adjunto livre-docente da FCMSCSP, São Paulo, SP, Brasil. 


\section{Introdução}

Gliomas são tumores cerebrais primários provenientes da proliferação descontrolada de células gliais, representadas pelos astrócitos, oligodendrócitos e células ependimárias. ${ }^{1} \mathrm{O}$ glioblastoma multiforme (GBM) assume notável importância ao representar pelo menos $80 \%$ dos gliomas de alto grau, ditos "malignos", que incluem aqueles classificados pela Organização Mundial de Saúde (OMS) como grau III (Astrocitoma Anaplásico, Oligodendroglioma Anaplásico, Oligoastrocitoma Anaplásico) ou grau IV (Glioblastoma, GBM), sendo, dessa forma, o tumor primário mais comum do sistema nervoso central (SNC), ${ }^{1,2}$

Anatomicamente, o GBM localiza-se principalmente na substância branca subcortical. ${ }^{3}$ As regiões mais comumente acometidas são: lobo temporal (31\%), parietal (24\%), frontal (23\%) e occipital (16\%). Mais raramente, lesões podem ser encontradas em região infratentorial e medula espinhal. ${ }^{3}$

Atribui-se o surgimento de glioblastomas à ocorrência de diferentes tipos de mutações. As mais frequentes são a perda do gene heterozigoto $(\mathrm{LOH})$ no braço do cromossomo $10 \mathrm{q}$ (encontrado em $60 \%$ a $90 \%$ dos casos) e a superexpressão do MDM2, vista em até $15 \%$ dos pacientes e associadas a um prognóstico ainda pior. Outras alterações importantes são a perda do cromossoma 10 do gene receptor do fator de crescimento epidérmico (EGFR) e mutações no gene p53, um gene supressor tumoral. $^{3}$

É crescente a importância do câncer no contexto global à medida que ocorre o envelhecimento da população associada a um estilo de vida que predispõe ao surgimento de câncer, tais como tabagismo, sedentarismo e alimentação. Assim, o objetivo do presente artigo é apresentar uma revisão sobre o GBM em idosos, com ênfase no seu tratamento cirúrgico, baseado em revisão da literatura atual.

\section{Discussão}

Do ponto de vista epidemiológico, estatísticas no âmbito global demonstram que aproximadamente 176.000 casos de câncer no SNC são diagnosticados anualmente, com mortalidade anual estimada em 128.000 óbitos, o que faz do câncer do SNC o responsável por aproximadamente $2 \%$ de todas as neoplasias malignas. ${ }^{4}$ No Brasil, segundo dados do Instituto Nacional de Câncer (INCA), são esperados, para o ano de 2012, 4.820 casos novos de câncer do SNC em homens e $4.450 \mathrm{em}$ mulheres, totalizando 9.270 casos. Esses valores correspondem a um risco estimado de cinco casos novos a cada 100 mil homens e quatro a cada 100 mil mulheres. ${ }^{5}$

Observa-se que, nas últimas décadas, a incidência dos tumores do SNC e a mortalidade decorrente deles aumentaram na maioria dos países desenvolvidos, principalmente nas faixas etárias mais avançadas. ${ }^{6}$ Estudos recentes apontam aumento de $5 \%$ ao ano na incidência de GBM em pacientes acima de 65 anos e aumentos ainda mais significativos (de 30\% para 254\%) na faixa etária acima dos 75 anos. ${ }^{3,6}$

Atribui-se esse fato não somente à introdução e à otimização de novas tecnologias diagnósticas menos invasivas, como tomografia computadorizada (TC) e ressonância magnética (RM), mas principalmente ao envelhecimento populacional. ${ }^{3,5,6}$

O GBM representa o tumor primário de SNC mais comum em idosos, e sua incidência demonstra crescimento progressivo especialmente nessa faixa etária. Sabe-se que o risco de desenvolver GBM aumenta com a idade, com uma taxa de incidência entre idosos (indivíduos com mais de 60 anos) de 17,5 casos por 100.000 pessoas/ano, o que confere um risco relativo de três a quatro vezes comparado a adultos jovens. Dessa forma, o manejo terapêutico do glioblastoma nesse subgrupo etário merece atenção especial não somente para o profissional neurocirurgião, mas, sobretudo, na elaboração das políticas de saúde pública. ${ }^{7,8}$

Os tratamentos do GBM disponíveis atualmente incluem a cirurgia na forma de ressecção total ou subtotal, radiocirurgia, radioterapia em suas formas standard (padrão) ou em cursos abreviados de curta duração, terapia antiangiogênica, terapia gênica, tratamento de suporte com agentes esteroides, além de quimioterapia com nitrosureia, bevacizumab e temozolomida, entre outros agentes quimioterápicos. ${ }^{7,9}$

\section{Modalidades de tratamento}

\section{Radioterapia}

A radioterapia é usualmente utilizada na abordagem terapêutica de pacientes idosos com diagnóstico de glioblastoma, seja na forma standard (60 Gy em 30 frações durante seis semanas) ou na forma de curta duração (por exemplo: 30 a 50 Gy em 6 a 20 frações durante duas a quatro semanas), mostrando vantagens sobre o tratamento de suporte paliativo. ${ }^{7}$

Estudo randomizado com 85 pacientes de idade igual ou superior a 70 anos, comparando radioterapia (50,4 Gy em 28 frações) acrescida de tratamento de suporte e tratamento de suporte apenas, demonstrou média de sobrevivência de 29,1 e 16,9 semanas para o primeiro e segundo grupos, respectivamente. ${ }^{10} \mathrm{Da}$ mesma forma, Marijnen et al., ${ }^{11}$ em estudo com 202 
pacientes entre 1990 e 2000, apontaram uma sobrevida consideravelmente maior nos pacientes submetidos à radioterapia em relação aos que não a receberam (10,6 versus 1,9 meses). É importante destacar ainda que, na maioria dos estudos, a presença de um bom estado neurológico e um Índice de Desempenho de Karnofsky (KPS, do inglês: Karnofsky Performance Status) de pelo menos 70 foram os fatores preponderantes como indicativos de melhores taxas de sobrevivência, com destaque especial ao KPS. ${ }^{?}$

Sabe-se que pacientes idosos têm maior suscetibilidade a sequelas neurocognitivas ocasionadas pela irradiação, mas poucos estudos conseguiram demonstrar isso de maneira significante. ${ }^{7,10}$ Assim, a dose ideal a ser utilizada nesses pacientes permanece incerta, além do fato de existirem dúvidas de que o tratamento convencional com 60 Gy realmente teria maior impacto na sobrevivência. ${ }^{7,10}$

Roa et al. ${ }^{12}$ em estudo prospectivo e randomizado envolvendo 100 pacientes tratados com radioterapia em dose convencional (60 Gy) ou com radioterapia de curso abreviado (40 Gy em 15 frações ao longo de três semanas), demonstraram médias de sobrevida em seis meses muito similares, com a vantagem de o grupo que recebeu o curso abreviado de radioterapia ter apresentado menores taxas de abandono prematuro do tratamento ( $10 \%$ versus $26 \%) .^{12}$

$\mathrm{Na}$ medida em que o tratamento do GBM na população idosa baseia-se na paliação, deve-se levar em conta a qualidade de vida ao escolher a modalidade de radioterapia a ser aplicada. Dessa maneira, em pacientes com KPS baixo, déficits neurológicos importantes e outros comemorativos de mau prognóstico, as terapias de curso abreviado oferecem claras vantagens. ${ }^{7,10}$

Sabe-se que a radioterapia é capaz de aumentar a sobrevida desses pacientes. Apesar de ser um ganho quantitativamente modesto, essa modalidade terapêutica torna-se estratégica, já que não traz significante queda no KPS, não interfere na qualidade de vida e não altera as funções cognitivas nesse subgrupo etário. ${ }^{7,10}$

\section{Quimioterapia}

A quimioterapia com utilização de nitrosureias mostrou-se capaz de aumentar a sobrevivência de pacientes idosos com glioblastoma em alguns estudos. Um estudo retrospectivo com 148 pacientes demonstrou média de sobrevivência de 43 semanas em pacientes de idade igual ou inferior a 60 anos e sobrevivência de 24 semanas em pacientes acima dos 60 anos, tendo ocorrência de sérias complicações mielossupressoras em $35 \%$ dos pacientes desse grupo etário. ${ }^{7}$
Brandes et al. ${ }^{13}$ relataram sobrevida de 12,7 meses em pacientes idosos com uso de procarbazina-lomustina-vincristina, com taxas de 6 e 12 meses de sobrevida de $90,6 \%$ e $56,2 \%$, respectivamente. No mesmo estudo, toxicidades hematológicas grau 3 foram observadas em até $20 \%$ dos participantes do estudo. ${ }^{13}$ Gilbert et al..$^{14}$ encontraram taxas de resposta ao tratamento de $76 \% \mathrm{e}$ sobrevida média de 11,9 meses em pacientes utilizando BCNU (carmustina) e cisplatina seguidos de radioterapia, mas novamente foram reportados importantes casos de toxicidade hematológica de graus 3 e 4 . $^{7}$

A temozolomida, mais recentemente introduzida para terapêutica de glioblastomas, é um agente alquilante imidazotetracênico com atividade antitumoral que sofre transformação química rápida em $\mathrm{pH}$ fisiológico, resultando no composto ativo MTIC (monometil-triaceno-imidazol-carboxamida). ${ }^{7}$ Sua propriedade lipofílica faz com que o medicamento atravesse a barreira hemoencefálica atingindo altas concentraçõesno liquor, o que facilita, assim, a ação sobre o tumor. ${ }^{7}$ Sua dosagem inicial é de $75 \mathrm{mg} / \mathrm{m}^{2}$ ao dia por seis semanas (42 dias), concomitante à radioterapia, seguida de administração adjuvante por seis ciclos, com doses de $150 \mathrm{mg} / \mathrm{m}^{2}$ no primeiro ciclo e $200 \mathrm{mg} / \mathrm{m}^{2}$ no segundo ciclo da fase adjuvante. $\mathrm{O}$ efeito citotóxico deriva da metilação de sítios específicos do DNA, mais especificamente da posição $\mathrm{O}^{6}$ da guanina. ${ }^{7}$

Chinot et al., ${ }^{15}$ em estudo feito com uso exclusivo com temozolomida, demonstraram que os pacientes idosos apresentaram 6,4 meses de sobrevida média e taxas de sobrevida, em 6 e 12 meses, de $60 \%$ e $25 \%$, respectivamente. Nesse mesmo estudo, metade dos pacientes conseguiu diminuir a dosagem de corticoides ou melhorar o KPS, embora toxicidade hematológica de graus 3 e 4 tenha sido relatada em $15 \%$ dos doentes. ${ }^{15}$ Achados semelhantes foram relatados por Glantz et al..$^{16}$ em estudo com temozolomida que encontrou sobrevida média de seis meses ( $11,9 \%$ em um ano) em pacientes acima de 70 anos, com mielossupressão como única toxicidade notificada, o que ocasionou redução ou ajuste da dose em $15 \%$ dos pacientes. ${ }^{16}$

De acordo com trial em fase II publicado em 2011, ${ }^{17}$ que analisa o uso de temozolomida em idosos sem utilização de radioterapia, pode-se encontrar progressão livre de doença de 16 semanas, sobrevida média de 25 semanas, melhora no KPS de 10 ou mais pontos em $33 \%$ dos indivíduos (com 26\% tornando-se capazes de cuidar de si próprios, isto é, KPS $\geq 70 \%$ ), exibindo, ainda, melhora global da qualidade de vida e cognição. ${ }^{17}$

De maneira geral, a temozolomida tem se mostrado como boa alternativa aos pacientes idosos com GBM e KPS menor que 70 , ao passo que exibe boa tolerância nesse subgrupo de doentes, demonstrando capacidade de melhora do estado funcional e aumentando a sobrevida. ${ }^{17}$ 


\section{Cirurgia}

O primeiro relato de ressecção de um glioma data de 1884, que foi realizada por Bennett e Godlee, em Londres. ${ }^{18}$ Desde então, a máxima sobrevida esperada após a ressecção de um GBM é de 16 a 18 meses, o que não difere muito dos índices atingidos há duas décadas, demonstrando a necessidade de avanços no tratamento desses tumores. ${ }^{19}$

Os objetivos da ressecção dos gliomas de alto grau incluem: alívio do efeito de massa, citorredução tumoral, obtenção do diagnóstico histopatológico, controle dos sintomas e tentativa de melhora nas taxas de sobrevida. ${ }^{7,19} \mathrm{O}$ alívio dos sintomas é percebido na redução da cefaleia, náuseas, vômitos e na melhora ou reversão dos déficits focais. Sabe-se que pacientes com melhoria dos sintomas após uso de esteroides tendem a apresentar maior benefício com a ressecção cirúrgica agressiva do tumor. ${ }^{20}$

Por outro lado, ressecções parciais podem exacerbar o edema tecidual e aumentar o risco de sangramentos no pós-operatório. A citorredução tumoral obtida com ressecções acima de $98 \%$ mostra-se benéfica na medida em que aumenta a eficácia das terapias adjuvantes, além de facilitar o diagnóstico histopatológico por oferecer maiores amostras do tecido. ${ }^{19}$

De acordo com os princípios da cirurgia oncológica, é de consenso que a ressecção tumoral total com margens livres é capaz de interferir na sobrevida do paciente. ${ }^{10}$ No entanto, na abordagem dos gliomas nem sempre isso é possível, pelo fato de que uma abordagem radical poderia ocasionar novos déficits neurológicos, piorando a morbidade e a qualidade de vida do paciente. ${ }^{10}$

Em estudos incluindo pacientes idosos e não idosos, concluiu-se que a ressecção de mais de $98 \%$ do volume tumoral é capaz de interferir na sobrevida, enquanto ressecções menores proporcionaram sobrevida semelhante à dos pacientes não abordados. ${ }^{10}$ Exemplo disso é o estudo retrospectivo realizado por Lacroix et al. ${ }^{21}$, em 2001, com 416 pacientes, que demonstrou sobrevida média de 13 versus 8,8 meses em pacientes com e sem ressecção cirúrgica superior a $98 \%$, respectivamente. Schneider et al., ${ }^{22}$ em estudo prospectivo com 31 pacientes, encontraram sobrevida média de 537 dias em pacientes submetidos à ressecção completa contra 237 dias naqueles que receberam ressecção incompleta. Stark et al., ${ }^{23}$ em estudo com 267 pacientes, também encontraram maiores taxas de sobrevida em pacientes com ressecções mais agressivas. ${ }^{10,22}$

No que diz respeito à abordagem cirúrgica de pacientes idosos, uma série de 30 pacientes com mais de 65 anos com diagnóstico de glioma maligno comparou a biópsia estereotáxica com a ressecção cirúrgica, encontrando sobrevida média de 85 e 171 dias ( $\mathrm{p}=0,035)$, respectivamente. ${ }^{24}$ Apesar da diferença encontrada, a época da deterioração neurológica não mostrou discrepância significativa entre os dois grupos. ${ }^{25} \mathrm{Da}$ mesma forma, estudo conduzido por Kelly e Hunt, ${ }^{25}$ em 1994, demonstrou um relativo prolongamento da sobrevida em pacientes submetidos à ressecção cirúrgica em comparação ao daqueles submetidos somente à biópsia estereotáxica (27 versus 15,4 semanas). ${ }^{25}$

Apesar de algumas evidências que apoiam a ressecção cirúrgica total, sabe-se que o caráter invasivo e infiltrativo dos gliomas de alto grau torna a ressecção curativa quase impossível por causa dos altos índices de recidiva nas bordas tumorais. ${ }^{10}$ Assim, mesmo abordagens radicais, como a hemiesferectomia, tentada por Walter Dandy em 1928 apud Keime-Guibert et al., ${ }^{10}$ não se mostraram resolutivas.

A máxima ressecção deve ser buscada objetivando os benefícios da cirurgia, mas tentando ao mesmo tempo preservar o estado neurológico do paciente. Observa-se que na maior parte dos casos, devido à citoarquitetura do GBM, a ressecção não proporciona incremento dos déficits prévios. Habitualmente, o esvaziamento tumoral é feito a partir do centro da lesão, onde estão as células com vascularização e metabolismo mais precários, estabelecendo-se limites visualmente e a partir da experiência do cirurgião. ${ }^{26}$ No entanto, deve-se ressaltar que a estimativa da ressecção por método intuitivo é pouco acurada. Métodos mais objetivos devem ser adotados, como a realização de TC e RM contrastadas dentro de 48 a 72 horas do pós-operatório. ${ }^{27}$

A fim de permitir a maior ressecção possível, obtendo-se o máximo benefício e reduzindo-se as sequelas e danos ao paciente, modernas técnicas são cada vez mais utilizadas. A ultrassonografia intraoperatória é uma técnica de baixo custo, fácil acesso e manuseio, que permite melhor avaliação da localização e extensão de lesões subcorticais. ${ }^{26}$ Outro método, a cirurgia guiada por fluorescência, consiste na administração de solução de ácido 5-aminolevulínico na dose de $20 \mathrm{mg} /$ $\mathrm{kg}$, que é carreado até as células tumorais pelo radical heme, resultando em protoporfirina IX fluorescente, cuja visualização é possível a partir de iluminação especial acoplada ao microscópio cirúrgico. ${ }^{28}$ Estudo comparando os resultados pós-operatórios de 139 pacientes que receberam ácido 5-aminolevulínico com 131 pacientes em que foi utilizada iluminação branca convencional demonstrou vantagem de $65 \%$ versus $36 \%$, respectivamente, na obtenção de ressecção completa. ${ }^{28}$ Esse mesmo estudo foi capaz de evidenciar $41 \%$ de progressão livre de doença após seis meses a favor do grupo que recebeu o ácido 5-aminolevulínico, contra apenas $21 \%$ no grupo controle, sem, entretanto, demonstrar frequência significativa de efeitos adversos entre os grupos. ${ }^{28}$

A neuronavegação, técnica em que se se utilizam imagens radiológicas pré-operatórias guiando a locali- 
zação e a ressecção cirúrgica, pôde se tornar ainda mais eficaz com a fusão de imagens anatômicas com imagens funcionais, associando-se também a tractografia. A principal crítica ao método é a possível mudança da localização das estruturas decorrente da manipulação cirúrgica, o que diminuiria a acurácia do método. Para suplantar tal inconveniente, tem-se associado a RM intraoperatória, gerando novas imagens durante a ressecção tumoral, o que pode adicionar até $28 \%$ na extensão da ressecção. ${ }^{26} \mathrm{~A}$ monitorização neurofisiológica que associa métodos como eletroencefalograma, potencial motor muscular, potenciais evocados e somatossensitivos, à medida que monitora continuamente a integridade da via neural, pode ser de grande valia na abordagem de gliomas em áreas eloquentes. ${ }^{26}$

Diversas séries e estudos foram capazes de demonstrar o efeito benéfico da ressecção cirúrgica nos pacientes com GBM em diversas faixas etárias. ${ }^{7,29,30}$ Entretanto, em faixas etárias avançadas o tema é cercado de controvérsias. A doença por si só exibe um curso desfavorável em idosos. As hipóteses para tal fato incluem: a menor tolerância aos procedimentos terapêuticos, a aumentada morbidade e mortalidade peri e pós-operatória, entre outros fatores como resistência à quimioterapia e à radioterapia, neurodegeneração, mutações genéticas e diferente padrão histológico. ${ }^{31}$

Devido ao comportamento desfavorável do GBM em idosos, o tratamento de pacientes nessa faixa etária nem sempre é realizado, e a inclusão desses pacientes em estudos e trials muitas vezes não ocorre, o que prejudica o surgimento de novas informações e a tomada de decisões baseada em evidências. Em decorrência da carência de evidências nessa população, acreditou-se, por muito tempo, que a idade era o principal fator prognóstico determinante..$^{31-33}$ Contrapondo o preconceito de se instituir um tratamento agressivo a pacientes idosos com GBM, estudos demonstraram que a idade é, de fato, um importante preditor de sobrevida em pacientes mais jovens. Porém, em idosos o principal fator determinante é o tratamento instituído, incluindo a ressecção total da lesão quando indicada. ${ }^{31,34}$

Estudo de coorte retrospectiva, publicado em 2008 por Mukerji et al.,$^{34}$ chega até mesmo a questionar a real validade de outros fatores prognósticos consagrados como a pontuação na escala de Karnofsky, duração dos sintomas, localização do tumor e presença de comorbidades. ${ }^{34}$ Artigo publicado em 2011 por Chaichana et al. ${ }^{35}$ foi capaz de identificar fatores pré-operatórios associados a menor sobrevida, como KPS menor que 80, tumores maiores que $4,0 \mathrm{~cm}$, presença de déficit motor ou de linguagem, déficit cognitivo e doença pulmonar obstrutiva crônica. ${ }^{35}$ Diversos estudos e trials publicados nos últimos anos focaram, quase unicamente, os aspectos relativos à quimioterapia e à radioterapia, mas pouco se estudou a respeito do grau de ressecção cirúrgica, suas indicações e seu impacto na sobrevida dos pacientes. ${ }^{31}$

Ewelt et al..$^{31}$ publicaram em 2011 um estudo que abordou a influência da ressecção cirúrgica na sobrevida de pacientes idosos com GBM. Nesse estudo foram incluídos 103 pacientes com GBM e idade superior a 65 anos, divididos em três grupos: um grupo em que os pacientes receberam somente tratamento cirúrgico (biópsia ou ressecção); outro grupo em que a cirurgia foi associada à radioterapia; e um último grupo em que a cirurgia foi associada a radioterapia e quimioterapia. Em todos os grupos comparou-se biópsia versus cirurgia citorredutora, sendo a mesma guiada por ácido 5-aminolevulínico. A sobrevida livre de progressão da doença e a sobrevida global foram, respectivamente, de 1,8/3,2/6,4 meses e 2,2/4,4/15,0 meses. A análise separada da sobrevida global média quanto à extensão da ressecção cirúrgica demonstrou para biópsia, ressecção parcial e ressecção completa, respectivamente, 2,2/7,0/13,9 meses. A sobrevida livre de progressão encontrada para cada modalidade cirúrgica foi de 10 meses para ressecção completa e 4,2 meses para ressecção parcial. Deve-se ressaltar, no entanto, que, no grupo que recebeu somente cirurgia e no grupo que recebeu cirurgia associada à radioterapia, a extensão da ressecção não se mostrou estatisticamente significativa na sobrevida global. ${ }^{31}$

Ainda, por meio desse estudo, foi possível observar que a decisão pela ressecção foi mais afetada pelo KPS $(>70 \%, p=0,01)$ do que pela idade. Até mesmo o fato de complicações pós-operatórias, como infecções do sítio cirúrgico e outros sistemas, meningite e fístula liquórica terem sido observadas em até $25 \%$ dos casos de GBM em idosos não deve interferir na decisão terapêutica, já que não foi possível demonstrar diferença significativa nas taxas de sobrevivência. ${ }^{31} \mathrm{O}$ estudo pôde concluir ainda que pacientes idosos podem obter o benefício máximo fornecido pela ressecção agressiva do tumor, sendo a extensão da ressecção o principal preditor prognóstico nessa faixa etária, sobretudo para pacientes tratados com radioterapia e quimioterapia. ${ }^{31}$

Como princípios gerais que orientam para uma abordagem cirúrgica mais conservadora ou mais extensa, há fatores como tamanho do tumor, sua profundidade e sua relação com áreas eloquentes ${ }^{27}$ (Tabela 1). Apesar de a decisão terapêutica invariavelmente ser influenciada pelo KPS e idade, pacientes idosos com GBM não devem, de forma alguma, ser excluídos da oportunidade de receber tratamento cirúrgico agressivo. ${ }^{31}$ Esses mesmos benefícios também haviam sido evidenciados em outros estudos previamente. . $5,36,37$

Em vista do surgimento desses novos conceitos aliado ao progressivo aumento da incidência do GBM na faixa etária acima dos 60 anos, tem-se necessidade 
crescente de se propor critérios bem estabelecidos de operabilidade que possam auxiliar na tomada de decisões ao conduzir pacientes nessa faixa etária, principalmente em casos de decisão acerca da realização de biópsia ou ressecção cirúrgica em pacientes idosos com KPS igual ou superior a 70\%. Dessa forma, o pontochave dessa discussão é o estabelecimento de critérios que auxiliem a identificar os pacientes idosos com GBM que possam se beneficiar de uma abordagem cirúrgica agressiva antes da terapia adjuvante. ${ }^{31}$

\begin{tabular}{ll}
\hline \multicolumn{1}{c}{$\begin{array}{c}\text { Tabela 1 - Princípios gerais de uma abordagem } \\
\text { cirúrgica conservadora ou extensa no GBM }\end{array}$} \\
\hline $\begin{array}{l}\text { Aspectos favoráveis à abordagem } \\
\text { cirúrgica conservadora }\end{array}$ & $\begin{array}{l}\text { Aspectos favoráveis à abordagem } \\
\text { cirúrgica agressiva }\end{array}$ \\
\hline $\begin{array}{l}\text { Tumores de crescimento } \\
\text { lento ou de história natural } \\
\text { não bem estabelecida }\end{array}$ & $\begin{array}{l}\text { História natural possivelmente } \\
\text { modificada pela ressecção cirúrgica }\end{array}$ \\
$\begin{array}{l}\text { Necessidade de minimizar } \\
\text { morbidades e hospitalização }\end{array}$ & $\begin{array}{l}\text { Indicação de ressecção por } \\
\text { razões práticas ou teóricas }\end{array}$ \\
\hline $\begin{array}{l}\text { Biópsia, especialmente se: } \\
\text { Ressecção extensa, } \\
\text { especialmente se: }\end{array}$ \\
$\begin{array}{l}\text { Tumor de pequeno volume } \\
\text { Tumor de grande volume }\end{array}$ \\
$\begin{array}{l}\text { Região eloção profunda } \\
\begin{array}{l}\text { Mínimo ou nenhum } \\
\text { efeito de massa }\end{array}\end{array}$ \\
$\begin{array}{l}\text { Paciente neurologicamente } \\
\text { assintomático }\end{array}$ & $\begin{array}{l}\text { Regiões não eloquentes } \\
\text { Adaptada de: Lacroix M, Abi-Said D, Fourney } \\
\text { A multivariate analysis of } 416 \text { patients with glioblastoma multiforme: prognosis, extent of } \\
\text { resection, and survival. J Neurosurg. 2001;95:190-8. }\end{array}$ \\
\hline
\end{tabular}

Tais achados são coerentes até mesmo do ponto de vista de saúde pública, já que estudos puderam concluir que, quando o ganho na sobrevida é proporcionalmente maior que o aumento da relação custo-efetividade, os custos por "anos de vida ganhos" diminuem, sendo a abordagem cirúrgica comparável e menos dispendiosa que outros tratamentos de primeira linha como a quimioterapia. $^{30,38}$

\section{Considerações gerais}

A decisão pelo tratamento cirúrgico do GBM no idoso envolve fatores como idade, KPS, presença de comorbidades, volume e localização tumoral e existência de déficits neurológicos à admissão. Por muito tempo a idade foi determinante na opção pelo tratamento e nos limites para se investir na terapêutica, mas pode-se concluir ao longo da revisão que a extensão da ressecção cirúrgica constitui-se o principal fator determinante do prognóstico, sobretudo para pacientes que foram eleitos para radioterapia e quimioterapia. Dessa forma, a idosos com KPS satisfatório, independente da idade, deve ser oferecido tratamento cirúrgico envolvendo ressecção completa da lesão.

\section{Referências}

1. Louis DN, Ohgaki H, Wiestler OD, Cavenee WK, editors. WHO Classification of tumors of the nervous system, editorial and consensus conference working group. Lyon: IARC Press; 2007.

2. Ironside JW, Moss TH, Louis DN, Weller RO. Diagnostic pathology of nervous system tumors. London: Churchill Livingstone; 2002.

3. Koul R, Dubey A, Torri V, Kakumanu A, Goyal K. Glioblastoma multiforme in elderly population. J Neurosurg. 2012;8(1):DOl:10.5580/2b2b. Disponível em: <http://yester. ispub.com/journal/the-internet-journal-of-neurosurgery/ volume-8-number-1/1881432761 glioblastoma-multiformein-elderly-population.html\#sthash. WJODb9ge.dpbs>. Acessado em: 08/06/2012.

4. Parkin DM. Global cancer statistics in the year 2000. Lancet Oncol. 2001;2(9):533-43.

5. Instituto Nacional de Câncer José Alencar Gomes da Silva. Estimativa 2012: incidência de câncer no Brasil. Rio de Janeiro: Inca; 2011.

6. Jemal A, Bray F, Center MM, Ferlay J, Ward E, Forman D. Global cancer statistics. CA Cancer J Clin. 2011;61(2):6990.

7. Lanzetta G, Minniti G. Treatment of glioblastoma in elderly patients: an overview of current treatments and future perspective. Tumori. 2010;96(5):650-8.

8. Brasil. Decreto-Lei $n^{\circ} 10.741$, de 1 de outubro de 2003. Dispõe sobre o Estatuto do Idoso e dá outras providências. Brasília: Diário Oficial da República Federativa do Brasil; 2003.

9. Thumma SR, Elaimy AL, Daines N, Mackay AR, Lamoreaux WT, Fairbanks RK, et al. Long-term survival after gamma knife radiosurgery in a case of recurrent glioblastoma multiforme: a case report and review of the literature. Case Rep Med. 2012;2012:545492.

10. Keime-Guibert F, Chinot O, Taillandier L, Cartalat-Carel S, Frenay M, Kantor G, et al. Radiotherapy for glioblastoma in the elderly. N Engl J Med. 2007;356(15):1527-35.

11. Marijnen CA, van den Berg SM, van Duinen SG, Voormolen $\mathrm{JH}$, Noordijk EM. Radiotherapy is effective in patients with glioblastoma multiforme with a limited prognosis and in patients above 70 years of age: a retrospective single institution analysis. Radiother Oncol. 2005;75(2):210-6.

12. Roa W, Brasher PM, Bauman G, Anthes M, Bruera E, Chan A, et al. Abbreviated course of radiation therapy in older patients with glioblastoma multiforme: a prospective randomized clinical trial. J Clin Oncol. 2004;22(9):1583-8.

13. Brandes AA, Vastola F, Basso U, Berti F, Pinna G, Rotilio $A$, et al. A prospective study on glioblastoma in the elderly. Cancer. 2003;97(3):657-62.

14. Gilbert M, O'Neill A, Grossman S, Grunnet M, Mehta $M$, Jubelirer $S$, et al. A phase II study of preradiation chemotherapy followed by external beam radiotherapy for the treatment of patients with newly diagnosed glioblastoma multiforme: an Eastern Cooperative Oncology Group study (E2393). J Neurooncol. 2000;47(2):145-52. 
15. Chinot OL, Barrie M, Frauger E, Dufour H, Figarella-Branger $\mathrm{D}$, Palmari J, et al. Phase II study of temozolomide without radiotherapy in newly diagnosed glioblastoma multiforme in an elderly populations. Cancer. 2004;100(10):2208-14.

16. Glantz M, Chamberlain M, Liu Q, Litofsky NS, Recht LD. Temozolomide as an alternative to irradiation for elderly patients with newly diagnosed malignant gliomas. Cancer. 2003;97(9):2262-6.

17. Gállego Pérez-Larraya J, Ducray F, Chinot O, Catry-Thomas I, Taillandier L, Guillamo JS, et al. Temozolomide in elderly patients with newly diagnosed glioblastoma and poor performance status: an ANOCEF phase II trial. J Clin Oncol. 2011;29(22):3050-5.

18. Bennett $\mathrm{H}$, Godlee RJ. Excision of a tumour from the brain. Lancet. 1884;2:1090-1.

19. Pang BC, Wan WH, Lee $\mathrm{CK}$, Khu KJ, Ng WH. The role of surgery in high-grade glioma - is surgical resection justified? A review of the current knowledge. Ann Acad Med Singapore. 2007;36(5):358-63.

20. Hentschel SJ, Lang FF. Current surgical management of glioblastoma. Cancer J. 2003;9(2):113-25.

21. Lacroix M, Abi-Said D, Fourney DR, Gokaslan ZL, Shi W, DeMonte F, et al. A multivariate analysis of 416 patients with glioblastoma multiforme: prognosis, extent of resection, and survival. J Neurosurg. 2001;95(2):190-8.

22. Schneider JP, Trantakis C, Rubach M, Schulz T, Dietrich J, Winkler D, et al. Intraoperative MRI to guide the resection of primary supratentorial glioblastoma multiforme - a quantitative radiological analysis. Neuroradiology. 2005;47(7):489-500.

23. Stark AM, Nabavi A, Mehdorn HM, Blömer U. Glioblastoma multiforme-report of 267 cases treated at a single institution. Surg Neurol. 2005;63(2):162-9.

24. Vuorinen V, Hinkka S, Färkkilä M, Jääskeläinen J. Debulking or biopsy of malignant glioma in elderly people - a randomised study. Acta Neurochir (Wien). 2003;145(1):5-10.

25. Kelly PJ, Hunt C. The limited value of cytoreductive surgery in elderly patients with malignant gliomas. Neurosurgery. 1994;34(1):62-6.

26. Maluf FC, Katz A, Corrêa S. Câncer do sistema nervoso central: tratamento multidisciplinar. São Paulo: Dendrix Edição e Design Ltda.; 2009.

27. Bernstein M, Berger MS. Neuro-oncology: the essentials. New York: Thieme Medical Publishers; 2000.

28. Stummer W, Pichlmeier U, Meinel T, Wiestler OD, Zanella $\mathrm{F}$, Reulen $\mathrm{HJ}$, et al. Fluorescence-guided surgery with 5-aminolevulinic acid for resection of malignant glioma: a randomised controlled multicentre phase III trial. Lancet Oncol. 2006;7(5):392-401.
29. Simpson JR, Horton J, Scott C, Curran WJ, Rubin P, Fischbach $\mathrm{J}$, et al. Influence of location and extent of surgical resection on survival of patients with glioblastoma multiforme: results of three consecutive Radiation Therapy Oncology Group (RTOG) clinical trials. Int J Radiat Oncol Biol Phys. 1993;26(2):239-44.

30. Laws ER, Shaffrey ME, Morris A, Anderson FA Jr. Surgical management of intracranial gliomas - does radical resection improve outcome? Acta Neurochir Suppl. 2003;85:47-53.

31. Ewelt C, Goeppert M, Rapp M, Steiger HJ, Stummer W, Sabel M. Glioblastoma multiforme of the elderly: the prognostic effect of resection on survival. $\mathrm{J}$ Neurooncol. 2011;103(3):611-8.

32. Sloan AE. Surgery for glioblastoma multiforme. J Neurosurg. 2011;114(3):585-6.

33. Barker FG 2nd, Chang SM, Larson DA, Sneed PK, Wara WM, Wilson $\mathrm{CB}$, et al. Age and radiation response in glioblastoma multiforme. Neurosurgery. 2001;49(6):128897.

34. Mukerji N, Rodrigues D, Hendry G, Dunlop PR, Warburton F, Kane PJ. Treating high grade gliomas in the elderly: the end of ageism? J Neurooncol. 2008;86(3):329-36.

35. Chaichana KL, Chaichana KK, Olivi A, Weingart JD, Bennett $\mathrm{R}$, Brem $\mathrm{H}$, et al. Surgical outcomes for older patients with glioblastoma multiforme: preoperative factors associated with decreased survival. Clinical article. J Neurosurg. 2011;114(3):587-94.

36. Stark AM, Hedderich J, Held-Feindt J, Mehdorn HM. Glioblastoma: the consequences of advanced patient age on treatment and survival. Neurosurg Rev. 2007;30(1):56-61.

37. Lebrun C, Fontaine D, Ramaioli A, Chamorey E, Bourg V, Paquis $P$, et al. Influence of surgery on survival of elderly patients with glioblastomas Neuro-Oncology. 2006;8:293372. Disponível em: <www.dukeupress.edu/neurooncology>. (DOI : 10.1215/15228517-2006-019).

38. Tengs TO. Cost-effectiveness versus cost-utility analysis of interventions for cancer: does adjusting for health-related quality of life really matter? Value Health. 2004;7(1):70-8.

\footnotetext{
Endereço para correspondência

Guilherme Brasileiro de Aguiar

Disciplina de Neurocirurgia,

Departamento de Cirurgia,

Faculdade de Ciências Médicas da

Santa Casa de Misericórdia de São Paulo

Rua Cesário Motta Jr., 112, Vila Buarque

01221-900 - São Paulo, SP, Brasil

Telefone: (55 11) 2176-7000

E-mail: guilhermebraguiar@yahoo.com.br
} 